\title{
Venous thromboprophylaxis in gastrointestinal bleeding
}

\author{
Neel Malhotra MD, Nilesh Chande MD FRCPC
}

$\mathrm{N}$ Malhotra, $\mathrm{N}$ Chande. Venous thromboprophylaxis in gastrointestinal bleeding. Can J Gastroenterol Hepatol 2015;29(3):145-148.

OBJECTIVE: To study the use of venous thromboembolism (VTE) prophylaxis and the incidence of thrombotic events in patients with acute gastrointestinal (GI) bleeding.

METHODS: Individuals admitted with a primary diagnosis of a GI bleed along with any endoscopically confirmed source (over a two-year period) were included. Patient comorbidity and data regarding anticoagulation or antiplatelet agent use before hospitalization were collected, in addition to type of VTE prophylaxis and duration of treatment. The primary end point was the development of VTE (deep vein thrombosis or pulmonary embolism) within one year of presentation.

RESULTS: Data from 504 patients admitted with GI bleeding were eligible for review. The total number of VTE events was 20 (4\%) while the mortality rate during hospitalization was $4.6 \%$; 397 patients were not given VTE prophylaxis during their hospitalization. Of the patients who were given VTE prophylaxis, 68 received prophylactic heparin or heparin derivatives during their admission. One hundred sixty-five patients had at least one other significant risk factor for VTE including recent or subsequent surgery, past thrombotic event or malignancy. The incidence of thrombosis in those with significant risk factors for VTE was significantly higher than those without $(8.5 \%$ versus $1.8 \% ; \mathrm{P}=0.0009)$. Overall, there was no significant difference in thrombotic events between individuals receiving pharmacological prophylaxis $(1.2 \%)$ and those who did not $(2.8 \%)(\mathrm{P}=0.4)$.

CONCLUSION: Overall, VTE prophylaxis did not significantly affect thrombotic events in patients admitted for an active GI bleed.

Key Words: Gastrointestinal bleeding; Venous thromboembolism prophylaxis

A dmissions for upper gastrointestinal (GI) bleeding in Canada account for 30 to 50 per 100,000 population per year (1). Patients presenting to hospital with GI bleeding often have an unpredictable clinical course, from being hemodynamically stable to requiring admission in the intensive care unit (ICU), emergency surgery or death. In Canada, the overall mortality rate for those found to have an upper GI bleed has been reoported to be $3.5 \%$ (1).

As a consequence of being hospitalized, all acutely ill patients, including those with GI bleed, are at increased risk for developing venous thromboembolism (VTE). The rate of these events in published studies varies between $1.0 \%$ and $1.9 \%$ (2). This is not a self-limiting problem because, once the diagnosis is made, future outcomes remain a concern. Pulmonary embolism (PE) alone is an independent risk factor for reduced survival within three months of onset, with a future risk for recurrence of VTE of up to $30 \%$ within 10 years (3). Up to $50 \%$ of VTEs are associated with right ventricular dysfunction, with the possibility of developing pulmonary hypertension (4). Patients hospitalized with GI bleeds fall within Virchow's triad due to long periods of immobility and venostasis.

As a result, there have been many studies showing the combined efficacy and safety of thromboprophylaxis in patients when clinically indicated (5). Most randomized controlled trials have studied low

\author{
La thromboprophylaxie veineuse en cas \\ d'hémorragie gastro-intestinale
}

OBJECTIF : Étudier l'utilisation de la prophylaxie de la thromboembolie veineuse (TEV) et l'évaluation du risque d'incidents thrombotiques chez les patients ayant une hémorragie gastro-intestinale (GI) aiguë.

MÉTHODOLOGIE : Les personnes hospitalisées en raison d'un diagnostic primaire d'hémorragie GI dont la source est confirmée par endoscopie (sur une période de deux ans) ont été incluses dans l'étude. Les chercheurs ont colligé la comorbidité des patients et les données relatives à l'anticoagulation ou à l'utilisation d'antiplaquettaires avant l'hospitalisation, ainsi que le type de prophylaxie de la TEV et la durée du traitement. Le critère d'évaluation primaire était l'apparition d'une TEV (thrombose veineuse profonde ou embolie pulmonaire) dans l'année suivant le rendez-vous.

RÉSULTATS : Les chercheurs ont examiné les données de 504 patients hospitalisés en raison d'une hémorragie GI. Au total, il y a eu 20 incidents de TEV (4\%), et le taux de décès pendant l'hospitalisation s'élevait à 4,6\%. De plus, 397 patients n'ont pas reçu de prophylaxie de la TEV pendant leur hospitalisation. Chez les patients sous prophylaxie, 68 ont reçu de l'héparine prophylactique ou des dérivés de l'héparine pendant leur hospitalisation. Cent soixante-cinq patients présentaient au moins un autre facteur de risque important de TEV, y compris une chirurgie récente ou ultérieure, des incidents thrombotiques passés ou un cancer. L'incidence de thrombose chez les personnes ayant d'importants facteurs de risque de TEV était considérablement plus élevée que chez celles qui n'en présentaient pas ( $8,5 \%$ par rapport à $1,8 \% ; \mathrm{P}=0,0009)$. Dans l'ensemble, le nombre d'incidents thrombotiques ne différait pas beaucoup entre les personnes sous prophylaxie pharmacologique $(1,2 \%)$ et celles qui ne l'étaient pas $(2,8 \%)(\mathrm{P}=0,4)$.

CONCLUSION : Dans l'ensemble, la prophylaxie de la TEV n'avait pas d'effet important sur les incidents thrombotiques chez les patients hospitalisés en raison d'une hémorragie GI.

molecular weight heparin (6) and new oral anticoagulants in reducing the risk of VTE in patients with acute medical illnesses (7). Naturally, the development of deep vein thrombosis (DVT) or PE leads to an increased burden on the health care system. In the United States alone, the economic impact of recurrent VTE was reflected in average total annual health care cost for primary diagnoses of $\$ 10,804$ per patient for DVT and $\$ 16,644$ per patient for PE (8).

In 2011, the American College of Physicians strongly recommended pharmacological prophylaxis for VTE in hospitalized medical patients unless the assessed risk for bleeding outweighed the likely benefits (9). As the medical community trends toward adhering to guidelines, withholding VTE prophylaxis in higher-risk individuals is largely unsupported by the literature (10). However, caution still needs to be exercised in those with evidence of a GI bleed. To better examine the complications of pharmacological prophylaxis in this population, a recent study involving patients hospitalized with lower GI bleeding was completed (9). While it did not show a statistically significant difference in mortality, it did show an increased number transfusions (mean $\pm[\mathrm{SD}] 3.47 \pm 2.36$ versus $2.56 \pm 2.37 ; \mathrm{P}=0.02$ ) and length of ICU stay $(6.07 \pm 3.87$ days versus $4.41 \pm 3.19$ days; $\mathrm{P}=0.02)$ with prophylactic subcutaneous heparin following admission. With some inherent risk and cost to the health care system, this raises the

\footnotetext{
Department of Medicine and Division of Gastroenterology, Western University/London Health Sciences Centre, London, Ontario

Correspondence: Dr Neel Malhotra, Department of Medicine, Western University, 800 Commissioners Road East, London, Ontario N6A 5W9.

Telephone 519-639-3055, e-mail neel.malhotra@londonhospitals.ca
}

Received for publication November 14, 2014. Accepted December 5, 2014 


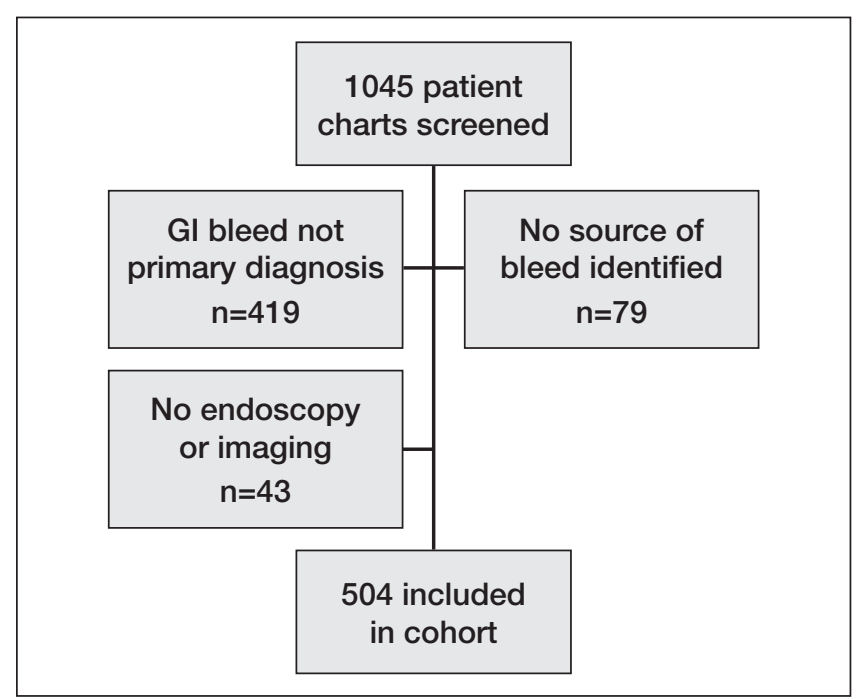

Figure 1) Chart review process. GI Gastrointestinal

\section{TABLE 1}

Demographic characteristics of the study group $(n=504)$

\begin{tabular}{lc}
\hline General characteristics & $70.5 \pm 15.3$ \\
Age, years, mean \pm SD & $5.5 \pm 7.3$ \\
Hospitalization, days, mean \pm SD & $154(30.6)$ \\
Upper gastrointestinal bleeding & $350(69.4)$ \\
Lower gastrointestinal bleeding & \\
Method of diagnosis & $350(69.4)$ \\
Gastroscopy & $146(30)$ \\
Colonoscopy & $5(1)$ \\
Red blood cell scan & $3(0.6)$ \\
Computed tomography scan & \\
Deep vein thrombosis prophylaxis & $107(21.2)$ \\
Yes & $397(78.8)$ \\
No & $23(4.6)$ \\
Death & $7(1.4)$ \\
Deep vein thrombosis prophylaxis & $16(3.2)$ \\
No deep vein thrombosis prophylaxis &
\end{tabular}

Data presented as $n(\%)$ unless otherwise indicated

question of whether this patient population is actually experiencing the perceived benefit of pharmacological DVT prophylaxis. Our aim was to conduct a retrospective cohort study to determine the overall use of VTE prophylaxis and the subsequent incidence of DVT/PE in patients admitted with a GI bleed.

\section{METHODS}

London Health Sciences Centre is a tertiary care centre located in London, Ontario. It operates two inpatient teaching hospitals and has a catchment of approximately two million in Southwestern Ontario. A retrospective chart review was performed on all inpatients admitted with a diagnosis of GI bleed between January 2007 and December 2008.

All patients $>18$ years of age were included, along with endoscopic evidence or radiological confirmation (computed tomography angiography or technetium-labelled red blood cell scan) of an active upper or lower GI bleed. Patients who developed evidence of bleeding while admitted to hospital for another reason were excluded. Only the initial event was considered in those with recurrent hospitalizations.

A standardized form was used to abstract data from electronic patient records. Demographic data, anticoagulation status, endoscopic findings and radiological evidence of VTE (DVT or PE) up to one year after discharge were recorded. This process was approved by Western University Research Ethics Board for Health Science Research Involving Human Subjects.
TABLE 2

Anticoagulation before admission

\begin{tabular}{lc}
\hline Antiplatelet & $186(57.4)$ \\
Acetylsalicylic acid & $12(3.7)$ \\
Clopidogrel & $44(13.6)$ \\
$\quad$ Combination & \\
Vitamin K antagonist & $36(11.1)$ \\
$\quad$ Warfarin & \\
Direct thrombin inhibitor & $3(0.9)$ \\
$\quad$ Dabigatran & $3(0.9)$ \\
Low molecular weight heparin & $2(0.6)$ \\
$\quad$ Dalteparin & \\
$\quad$ Enoxaparin & $37(11.4)$ \\
Combination & $1(0.3)$ \\
$\quad$ Antiplatelet agent + vitamin K antagonist & \\
Low molecular weight heparin + vitamin K antagonist & \\
\hline Data presented as $n$ (\%) &
\end{tabular}

Demographic data comprised patient age in additon to information regarding comorbidities that may have contributed to a thrombotic event including known history of thrombotic events, recent surgery (within three months), and diagnosis of malignancy or cirrhosis. Documenting anticoagulation status included antiplatelet agents, vitamin $\mathrm{K}$ antagonists, direct thrombin inhibitors, as well as heparin and heparin derivatives at the time of admission while hospitalized and after discharge. Any of these agents were considered to be protective against the development of thrombosis even if they were discontinued for a portion of the hospital stay.

The primary outcome was the incidence of VTE up to one year after discharge based those who received anticoagulant therapy for prophylaxis against VTE during hospitalization. The potential role of hypercoagulable states (malignancy, cirrhosis, recent surgery and past thrombosis) as risk factors for thromboembolic disease was used as a secondary outcome.

\section{Statistical analysis}

Standard descriptive analysis was performed to determine the source of GI bleeding along with the modality of endoscopic intervention most used for diagnosis. The frequency of VTE development, in additon to anticoagulation and hypercoagulable states, was also documented. Fisher's exact test with two-tailed P value using SAS/Stat Software version 9.1.3 (SAS Institute Inc, USA) was performed to analyze the primary outcome of VTE in those who received pharmacological prophylaxis comapred with those who did not. A subgroup analysis was performed to investigate the outcome of pharmacological prophylaxis in those with significant risk factors likely predisposing to thromboembolic complications.

\section{RESULTS}

After initial review, 1045 potential charts were identified. Of these, 419 had a primary diagnosis other than GI bleed leading to admission (Figure 1). Further screening found that 43 patients had no endoscopic procedure or imaging to confirm their diagnosis. Seventy-nine investigations were completed, but no source of bleeding was identified. This left 504 patient charts eligible for the present study. The demographic characteristics of the study group are summarized in Table 1. A higher number of patients were found to have a lower GI bleed (69.4\%) compared with an upper GI bleed source (30.6\%). In addition, pharmacological prophylaxis against VTE was only given in a minority $(21.2 \%)$ of cases.

Table 2 summarizes the distribution of anticoagulant and antiplatelet agents before admission. The vast majority of patients were on some form of antiplatelet therapy (64.7\%), with acetylsalicylic acid the most common agent $(57.4 \%)$. For patients who were started on some form of VTE prophylaxis, the most common agents were heparin or low molecular weight heparin, as shown in Table 3. It should also be 
TABLE 3

\begin{tabular}{lc} 
Pharmacological anticoagulation during hospital stay \\
\hline Antiplatelet \\
Acetylsalicylic acid & $18(16.8)$ \\
Clopidogrel & $5(4.7)$ \\
Combination & $6(5.6)$ \\
Vitamin K antagonist & \\
$\quad$ Warfarin & $3(2.8)$ \\
Direct thrombin inhibitor & \\
$\quad$ Dabigatran & $0(0)$ \\
Heparin or low molecular weight heparin & \\
Dalteparin & $48(44.9)$ \\
Enoxaparin & $1(0.9)$ \\
Heparin & $19(18.7)$ \\
Combination & \\
Antiplatelet agent + vitamin K antagonist & $0(0)$ \\
Antiplatelet agent + low molecular weight heparin & $4(3.7)$ \\
Antiplatelet agent + heparin & $3(2.8)$
\end{tabular}

Data presented $n$ (\%)

noted that some patients were continued on their antiplatelet or anticoagulant medication while being treated.

Table 4 lists additional risk factors that played a role in the development of thromboembolic complications. Of these, history of malignancy was the most common $(n=83)$ followed by evidence of cirrhosis $(n=45)$. Less frequent risk factors included history of past thrombotic events $(n=13)$ and recent surgery (within the past three months) $(n=7)$.

Overall, the incidence of VTE in the present study was 4\%. Using the Fisher's exact test, the overall incidence of DVT/PE was significantly higher in those with higher-risk conditions $(\mathrm{P}=0.0009)$. As shown in Table 5, the combined high/low risk group had a reduced incidence of thromboembolic complications (1.2\%) with pharmacological prophylaxis, although not statistically significant $(\mathrm{P}=0.4)$. This difference was also observed in the subgroup of individuals with comorbidities, with the incidence of VTE being $1.8 \%$.

\section{DISCUSSION}

It has been well established that acutely ill medical patients benefit from pharmacological prophylaxis for VTE (11). The difficulty in patients with evidence of GI bleed is the concern for recurrent bleeding and hemodynamic stability. Adding any antiplatelet or anticoagulant in these situations often increases concern among health care professionals, which was reflected in the present study by the low number of individuals who were given VTE prophylaxis $(n=107)$. Interestingly, current evidence shows that there is no increase in mortality among patients with lower GI bleeding treated with VTE prophylaxis (10). However, there was a longer ICU stay and increased number of transfusions only within the first $24 \mathrm{~h}$ after admission (10). In a study investigating the clinical outcomes of patients with a recent diagnosis of VTE, $40 \%$ experienced a GI bleed 30 days previously (12). Three-month mortality was as high as $23 \%$, which is a clinically important measure, indicating the importance of VTE prophylaxis.

Importantly, our study showed that thromboembolic complications do exist in patients admitted with a GI bleed. VTE is significantly more common among individuals with other risk factors predisposing them to hypercoagulable states $(\mathrm{P}=0.0009)$. Although not a statistically significant difference, we were also able to demonstrate that VTE prophylaxis decreased the incidence of thromboembolic events compared with those who did not receive it $(1.2 \%$ versus $2.8 \%$, respectively; $\mathrm{P}=0.4$ ).

Other observational studies have reported that a large percentage of individuals with VTE were $>65$ years of age and had additional risk factors, including malignancy, for VTE (12). Patients in our study had a mean age of 70.5 years, with a mean length of hospitalization of 5.5 days. The most frequent comorbidities believed to confer additional risk for

\section{TABLE 4}

Comorbidities of patients who developed thrombotic conditions $(n=165)$

\begin{tabular}{ll}
\hline Comorbidity & \\
Malignancy & 83 \\
Past thromboembolic events* $^{*}$ Recent surgery $^{\dagger}$ & 13 \\
Cirrhosis & 7 \\
Combination & 45 \\
Malignancy - cirrhosis & \\
Malignancy - past thromboembolic events & 11 \\
Malignancy - surgery & 5 \\
\hline
\end{tabular}

Data presented as $n$. *Includes pulmonary embolism, deep vein thrombosis, aortic clot, apical thrombus and portal vein thrombosis; ${ }^{\dagger}$ Within three months

TABLE 5

Incidence of thromboembolic disease (deep vein thrombosis [DVT]/pulmonary embolism [PE])

\begin{tabular}{lccc}
\hline & $\begin{array}{c}\text { Incidence of } \\
\text { DVT/PE, \% }\end{array}$ & P & OR (95\%Cl) \\
\hline All patients ( $\mathrm{n}=504)^{*}$ & 4.0 & & \\
$\quad$ No comorbidities & 1.8 & & \\
$\quad$ Comorbidities & 8.5 & 0.0009 & $5.1(1.9-13.6)$ \\
Comorbidities ( $\mathrm{n}=165)$ & & & \\
$\quad$ DVT prophylaxis & 1.8 & & \\
$\quad$ No prophylaxis & 6.7 & 0.72 & $0.7(0.2-3.0)$ \\
$\quad$ No comorbidities ( $\mathrm{n}=339)$ & & & \\
$\quad$ DVT prophylaxis & 0.8 & & \\
$\quad$ No prophylaxis & 0.8 & 0.11 & $0.2(0.05-1.3)$ \\
Comorbidities + no comorbidities & & & \\
$\quad$ DVT prophylaxis & 1.2 & & \\
$\quad$ No DVT prophylaxis & 2.8 & 0.4 & $0.6(0.2-1.6)$ \\
\hline *Includes both with and without $D V T$ prophylaxis &
\end{tabular}

thromboembolism included malignancy $(n=83)$, cirrhosis $(n=45)$ and past thrombotic events $(n=13)$.

Low-dose acetylsalicylic acid has been associated with an increased risk for upper GI bleeding (13). Our data indicate that antiplatelet therapy, specifically acetylsalicylic acid, was used most commonly $(57.4 \%)$ by patients before presenting with upper or lower GI bleeding. Baseline demographic data showed that lower GI bleeds (69.4\%) were more common than upper GI bleeds (30.6\%). This supports the higher risk associated with acetysalicylic acid therapy in lower GI bleeds documented in a recent prospective study (14).

The lack of a significantly lower incidence of VTE observed in our cohort was likely multifactorial. First, there were low event rates observed in both groups. The study period could have been extended to determine the longer-term incidence of DVT/PE. Second, our teaching centre has a large catchment area with a significant referral base. Confirmation of thromboembolic events required documented radiological evidence and, for patients transferred back to their local hospital, some would be lost to follow-up, which may have led to an underestimation of our event rates.

Overall, it would be our recommendation to implement VTE prophylaxis on a case-by-case basis for patients admitted with an active GI bleed, particularly if they have other factors predisposing them to thrombosis. Further studies investigating complications of low-dose anticoagulant therapy are likely needed to adequately quantify the risk associated with therapy and to reassure future practice.

DISCLOSURES: The authors have no financial disclosures or conflicts of interest to declare. 


\section{REFERENCES}

1. Targownik LE, Nabalamba A. Trends in management and outcomes of acute nonvariceal upper gastrointestinal bleeding: 1993-2003. Clin Gastroenterol Hepatol 2006;4:1459-66.

2. Guijarro R, San Roman C, Arcelus JI, et al. Bleeding and venous thromboembolism arising in acutely ill hospitalized medical patients. Findings from the Spanish National Discharge Database. Eur J Intern Med 2014;25:137-41.

3. Heit JA. Venous thromboembolism: Disease burden, outcomes and risk factors. J Thromb Haemost 2005;3:1611-7.

4. Kearon C. Natural history of venous thromboembolism. Circulation 2003;107:I22-I30.

5. Dentali F, Douketis JD, Gianni M, Lim W, Crowther MA. Meta-analysis: Anticoagulant prophylaxis to prevent symptomatic venous thromboembolism in hospitalized medical patients. Ann Intern Med 2007;146:278-88.

6. Samama MM, Cohen AT, Darmon JY, et al. A comparison of enoxaparin with placebo for the prevention of venous thromboembolism in acutely ill medical patients. Prophylaxis in Medical Patients with Enoxaparin Study Group. N Engl J Med 1999;341:793-800.

7. Roussin A. Effective management of acute deep vein thrombosis: Direct oral anticoagulants. Int Angiol June 13, 2014 (Epub ahead of print).

8. Spyropoulos AC, Lin J. Direct medical costs of venous thromboembolism and subsequent hospital readmission rates: An administrative claims analysis from 30 managed care organizations. J Manag Care Pharm 2007;13:475-86.
9. Qaseem A, Chou R, Humphrey LL, Starkey M, Shekelle P. Venous thromboembolism prophylaxis in hospitalized patients: A clinical practice guideline from the American College of Physicians. Ann Intern Med 2011;155:625-32.

10. Deutsch GB, Kandel AR, Knobel D, et al. Bleeding risk secondary to deep vein thrombosis prophylaxis in patients with lower gastrointestinal bleeding. J Intensive Care Med 2012;27:379-83.

11. Kahn SR, Lim W, Dunn AS, et al. Prevention of VTE in nonsurgical patients. In: Antithrombotic Therapy and Prevention of Thrombosis, 9th edn: American College of Chest Physicians Evidence-Based Clinical Practice Guidelines. Chest 2012;141:e195S-e226S

12. Nieto JA, Camara T, Gonzalez-Higueras E, et al. Clinical outcome of patients with major bleeding after venous thromboembolism. Findings from the RIETE Registry. Thromb Haemost 2008;100:789-96

13. Valkhoff VE, Sturkenboom MC, Hill C, Veldhuyzen van Zanten S, Kuipers EJ. Low-dose acetylsalicylic acid use and the risk of upper gastrointestinal bleeding: A meta-analysis of randomized clinical trials and observational studies. Can J Gastroenterol 2013;27:159-67.

14. Huang ES, Strate LL, Ho WW, Lee SS, Chan AT. A prospective study of aspirin use and the risk of gastrointestinal bleeding in men. PLoS One 2010;5:e15721. 


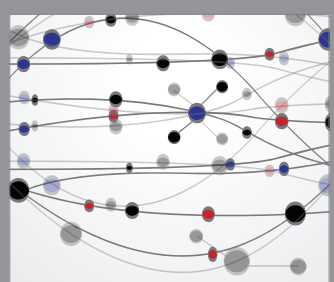

The Scientific World Journal
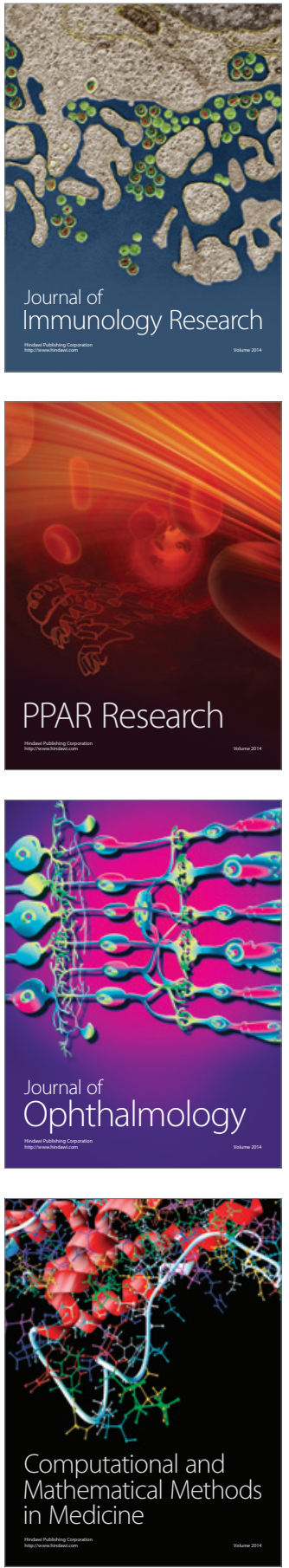

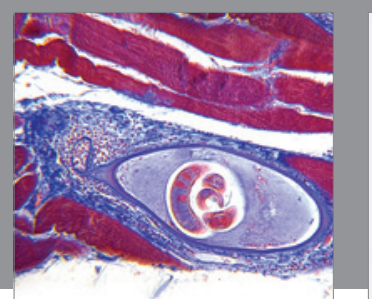

Gastroenterology Research and Practice

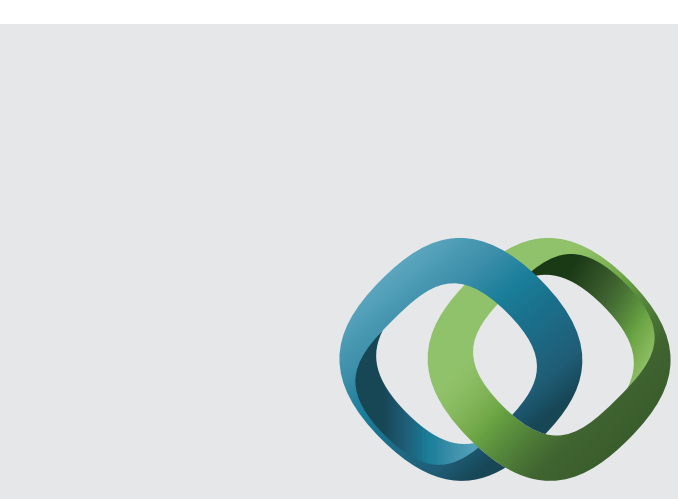

\section{Hindawi}

Submit your manuscripts at

http://www.hindawi.com
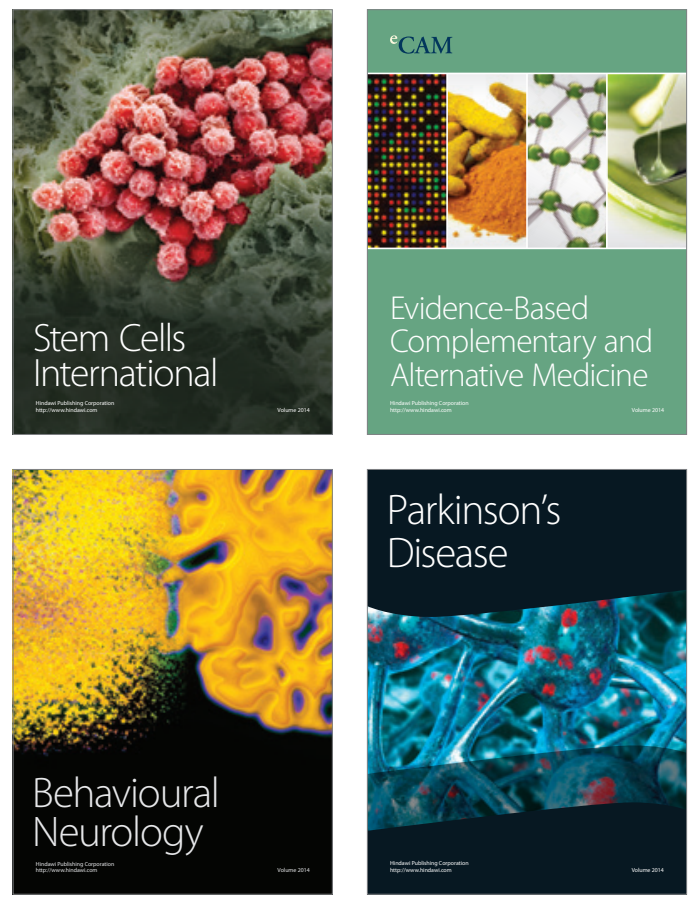
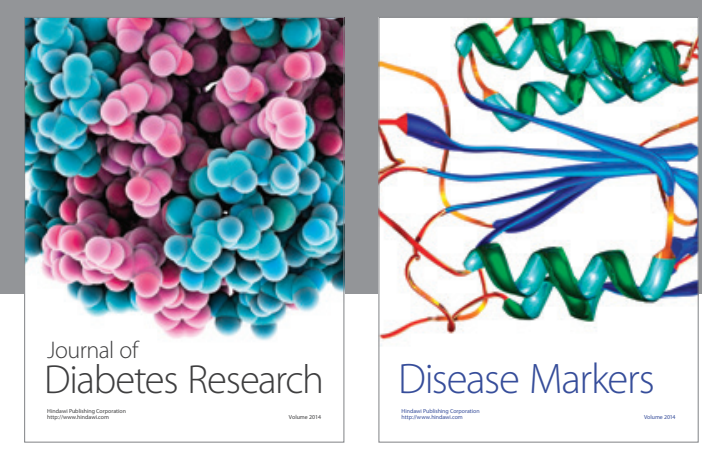

Disease Markers
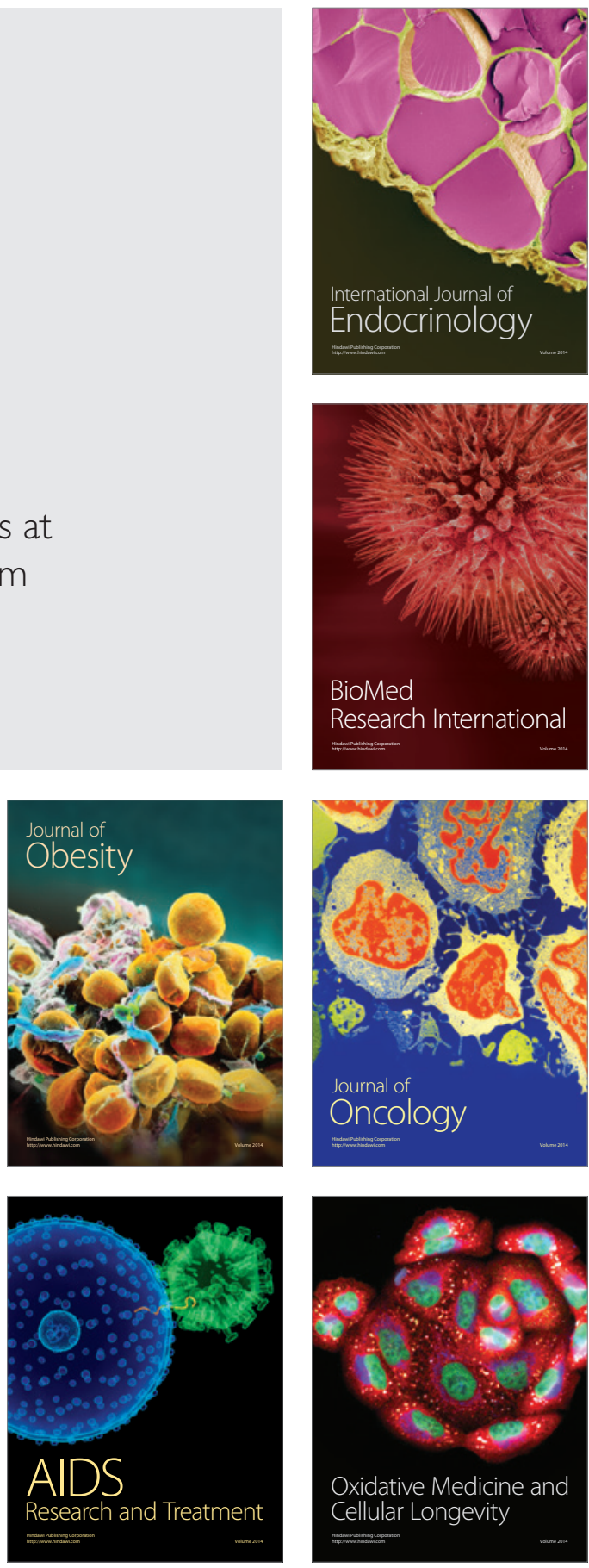